\title{
First detection and genomic characteristics of bovine torovirus in dairy calves in China
}

\author{
Hao $\mathrm{Li}^{1} \cdot$ Bin Zhang ${ }^{1,2} \cdot$ Hua Yue $^{1} \cdot$ Cheng Tang $^{1,2}(\mathbb{D}$
}

Received: 15 January 2020 / Accepted: 12 April 2020 / Published online: 9 May 2020

C) Springer-Verlag GmbH Austria, part of Springer Nature 2020

\begin{abstract}
Bovine torovirus (BToV) is a diarrhea-causing pathogen. In this study, 92 diarrheic fecal samples from five farms in four provinces in China were collected and tested for BToV using a RT-PCR assay, and $21.73 \%$ samples were found to be BToV positive. Moreover, two complete BToV genome sequences (MN073058 and MN073059) were obtained from the clinical samples, which were 28,297 and 28,301 nucleotides in length, respectively. Sequence analysis showed that the two isolates shared 10 identical amino acid mutations in the $\mathrm{S}$ protein compared to the complete $\mathrm{S}$ sequences of BToV available in the GenBank database. In addition, seven consecutive amino acid mutations were found from aa 1,486 to 1,492 in the $\mathrm{S}$ protein of isolate MN073058. Moreover, the two isolates shared one identical amino acid mutation in the receptor binding sites of the HE protein. To the best of our knowledge, this is the first report on the epidemic and genomic characterization of BToV in China, which is helpful for further understanding the genetic evolution of BToV.
\end{abstract}

\section{Introduction}

Toroviruses are members of the family Coronaviridae, order Nidovirales, and include bovine torovirus (BToV) [1, 2], Berne virus (EToV) [3], porcine torovirus (PToV) [4], and human torovirus (HToV) [5]. BToV mainly causes diarrhea in calves and adult cattle. The virus can not only be detected in feces but also in the respiratory tract, indicating that the virus has dual tissue tropism [6-9]. BToV has been detected in 16 countries with a wide geographical distribution [2, 7, 10-19]. In addition, a sequence reported to be from goat torovirus is present in the GenBank database, and in 2012, researchers detected the presence of PToV in pig herds by RT-PCR in China [20].

Handling Editor: Sheela Ramamoorthy.

Electronic supplementary material The online version of this article (https://doi.org/10.1007/s00705-020-04657-9) contains supplementary material, which is available to authorized users.

Cheng Tang

tangcheng101@163.com

1 College of Life Science and Technology, Southwest Minzu University, No. 16, South 4th Section 1st Ring Road, Chengdu 610041, China

2 Innovation Team of Beef Cattle, Chengdu, Sichuan, China
Presently, three BToV genomic sequence (AY427798, LC088094, LC088095) can be obtained from the GenBank database. The BToV genome encodes four structural proteins: spike glycoprotein (S), membrane glycoprotein (M), hemagglutinin esterase (HE), and nucleocapsid glycoprotein (N) [21]. The S protein is involved in viral infectivity and induces the production of neutralizing antibodies [22]. The $\mathrm{M}$ protein plays a role in $\mathrm{BToV}$ assembly and nucleocapsid recognition [21]. The HE has a putative F-G-D-S motif and has acetylesterase activity specific for N-acetyl-9-Oacetylneuraminic acid $[1,21]$. HE contains three functional domains: a lectin domain (R), an esterase domain (E) and a membrane-proximal domain (MP) [23]. The N protein is the only viral RNA-binding polypeptide found in infected cells. It protects the genome and ensures its timely replication and reliable transmission, as well as playing a role in virus transcription and translation [24, 25].

Diarrhea is a common disease in dairy cows in China, which leads to serious economic losses. Bovine coronavirus (BCoV), bovine group A rotavirus (BRVA), bovine viral diarrhea virus (BVDV), bovine norovirus (BNoV), and nebovirus $(\mathrm{NeV})$ had been identified as common diarrheacausing viruses in bovines in China [26-30]. However, there is currently no information regarding BToV in China. The goal of this study was to detect and the genome of characterize of BToV in calves in China. 


\section{Materials and methods}

\section{Sample collection}

From September to December 2018, 92 diarrheic fecal samples were collected from calves (aged $<3$ months) from five farms in four provinces of China. These included 23 samples from Sichuan Province (one farm), 39 from Liaoning Province (two farms), 20 from Shanxi Province (one farm), and 10 from Henan Province (one farm). All samples were stored at $-80{ }^{\circ} \mathrm{C}$ in sterile $50-\mathrm{mL}$ centrifuge tubes until further testing.

\section{RNA extraction and CDNA synthesis}

Total RNA from $300 \mu \mathrm{L}$ of the fecal suspension was extracted using a QIAamp Viral RNA Mini Kit (QIAGEN, Hilden, Germany) according to the manufacturer's instructions. Complementary DNA (cDNA) was synthesized using a Primescript ${ }^{\mathrm{TM}}$ reverse transcription kit (TaKaRa, Dalian, China) and stored at $-20{ }^{\circ} \mathrm{C}$.

\section{Screening for BToV by RT-PCR}

BToVs were detected using a specific RT-PCR assay established in our laboratory. The specificity and reproducibility of the RT-PCR assay has been validated, and the detection limit is $1.908 \times 10^{-1} \mathrm{pg} / \mu \mathrm{L}$. Briefly, a pair of primers $\left(5^{\prime}-\right.$ GTACTAWTTTTCCAGCTYTGC-3' and 5' -CCAACA CAAATCCGCAAYGC-3') was used to amplify a 406-bp fragment of the $M$ gene (position 25,755-26,160 bp of the BToV Ishikawa/2010 genomic sequence, GenBank accession number LC088094.1). The amplification was conducted in a $25-\mu \mathrm{L}$ reaction volume containing $0.05 \mu \mathrm{M}$ forward primer, $0.05 \mu \mathrm{M}$ reverse primer, $2 \mu \mathrm{L}$ of cDNA, 12.5 $\mu \mathrm{L}$ of EmeraldAmp PCR Master Mix $(2 \times$ Premix $)$ (TaKaRa Bio Inc.), and an appropriate volume of doubledistilled water. The RT-PCR amplification products were sequenced directly in both directions by Sangon Biotech (Chengdu, China) to verify the accuracy of detection.

\section{Screening for coinfection with major bovine enteric viruses}

To investigate coinfection with BRVA, BCoV, BVDV, NeV and BNoV, all BToV-positive samples were subjected to previously described specific RT-PCR assays for these viruses. [27-29, 31] The detection of BRVA, BCoV, NeV were following our previous reports, BVDV was following
Table 1 BToV coinfection with major enteric viruses

\begin{tabular}{lc}
\hline Enteric pathogens & $\begin{array}{c}\text { Positive } \\
\text { rate }(\%)\end{array}$ \\
\hline BToV (only) & 40 \\
BToV + BCoV & 5 \\
BToV + BRVA & 5 \\
BToV + BCoV + BRV & 5 \\
BToV + BCoV + BVDV & 10 \\
BToV + BRVA + NeV & 10 \\
BToV + BRVA + BNoV & 10 \\
BToV + BRVA + BVDV + BNoV & 5 \\
BToV + BCoV + BVDV + BNoV + NeV & 5 \\
BToV + BRVA + BVDV + BNoV + NeV & 5 \\
\hline
\end{tabular}

the previous report [], BNoV was following the previous report [].

\section{Amplification of the complete genome}

To investigate the genomic characteristics of BToV isolates from China, 29 primer pairs were designed to amplify their genomes (Table S1). The 3' and 5' ends of the viral genome were sequenced by rapid amplification of cDNA ends using a Smart RACE cDNA Amplification Kit (TaKaRa). The PCR products were cloned into the pMD19-T simple vector (TaKaRa) for further sequencing (Sangon Biotech).

\section{Sequence, phylogeny, and recombination analysis}

Sequences were assembled using SeqMan software (version 7.0; DNASTAR, Inc., WI, USA). Putative ORFs in the linear genomes were identified using the ORF Finder tool (http://www.ncbi.nlm.nih.gov/gorf/gorf.html). Nucleotide and deduced amino acid sequences were compared using the MegAlign program of Lasergene software, version 7.1 (DNASTAR, Madison, WI, USA). A multiple sequence alignment was performed, and a neighbor-joining phylogenetic tree was built using the interior branch test method with the aid of MEGA7 software. Possible recombination events were identified using SimPlot software (version 3.5.1) and the Recombination Detection Program (RDP 4.95) by the RDP, Chimaera, BootScan, 3Seq, GENECONV, MaxChi, and SiScan methods.

\section{Results}

\section{BToV detection and coinfections}

Among the 92 diarrheic samples, 21.73\% (20/92) tested BToV-positive by RT-PCR. The detection rate was $69.56 \%$ 
(16/23), 5.12\% (2/39) and 10\% (2/20) in Sichuan, Liaoning, and Shanxi province, respectively. BToV was not found in diarrhea samples from Henan province. Twelve were coinfected with BCoV, BRVA, BVDV, BNoV or NeV (Table 1).

\section{Molecular characterization of partial M genes}

A portion of the $\mathrm{M}$ gene of 20 positive samples was sequenced, and all partial sequences of the $\mathrm{M}$ gene (GenBank accession no. MN073058-MN073079) were submitted to the GenBank database. The 20 nucleotide sequences were $98.8 \%$ to $100 \%$ identical to one another and $96.3 \%$ to $99.3 \%$ identical to those of previously reported BToV strains. A phylogenetic tree was constructed using the 406bp $\mathrm{M}$ fragment sequence, including the $20 \mathrm{BToV}$ sequences from calves as well as 10 other BToV sequences from the GenBank database. The $20 \mathrm{BToV}$ sequences from this study were divided into two different groups (Fig. S1). One of these groups included BToV isolates from three different provinces, which formed a unique large branch, but the one remaining strain clustered with the Turkish strains HT1 and HT2.

\section{Overview of the characteristics of the two complete BToV genome sequences}

To obtain more-precise information about the evolutionary relationships of the $\mathrm{BToV}$ isolates from calves, six representative $\mathrm{BToV}$ isolates were chosen from four positive farms, and two BToV isolates were chosen from each province. However, we were only able to successfully assemble two complete genomic sequences (SC-1 Sichuan / 2018 and SC-2 Sichuan / 2018), both of which were from the same farm in Sichuan province. These sequences were submitted to the GenBank database with accession numbers MN073058 and MN073059. The length of the linear genome was 28,297 and $28,301 \mathrm{nt}$, respectively, and the $\mathrm{G}+\mathrm{C}$ content was $36.36 \%$. The complete genome of the strains shared 42.9-99.2\% nt sequence identity with the 10 genome sequences of mammalian ToVs in the GenBank database. Analysis of the complete genome sequences revealed that the two Chinese BToVs shared $99.1 \%$ nucleotide sequence identity with each other, $96.89 \%$ to $97.07 \%$ identity with the two genome sequences from Japan, and shared $81.9 \% \mathrm{nt}$ identities with the prototype strain Bread 1 (Fig. 1).

\section{Complete S gene sequences of strains SC-1 and SC-2}

The complete S genes of strains SC-1 and SC-2 were both $4,755 \mathrm{bp}$ in length and encoded a protein of 1,586 amino acids. The complete $\mathrm{S}$ genes shared $99.7 \%$ nt sequence identity and $99.3 \%$ aa sequence identity with each other and shared $92.2-97.6 \%$ nt sequence identity (91.3-98.4\% aa

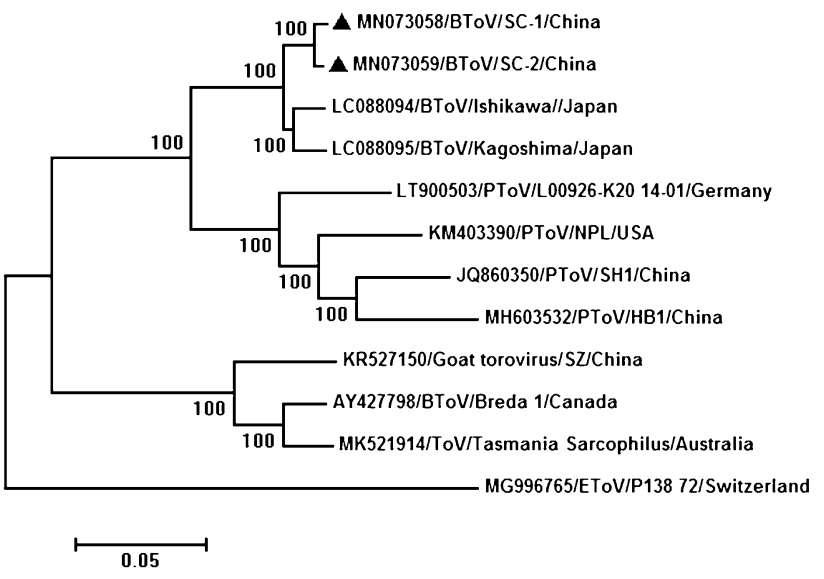

Fig. 1 Phylogenetic tree based on the complete genomic nucleotide sequence of all mammalian toroviruses. Sequence alignments were performed using ClustalW in MEGA 7.0 software. The tree was constructed by the maximum-likelihood method with bootstrap values calculated for 1000 replicates. The scale bar indicates amino acid substitutions per site. Bovine torovirus was found in calves and may be related to members of the genus Coronavirus. The bovine torovirus strains BToV/SC-1/China and BToV/SC-2/China investigated in this study are indicated by black triangles

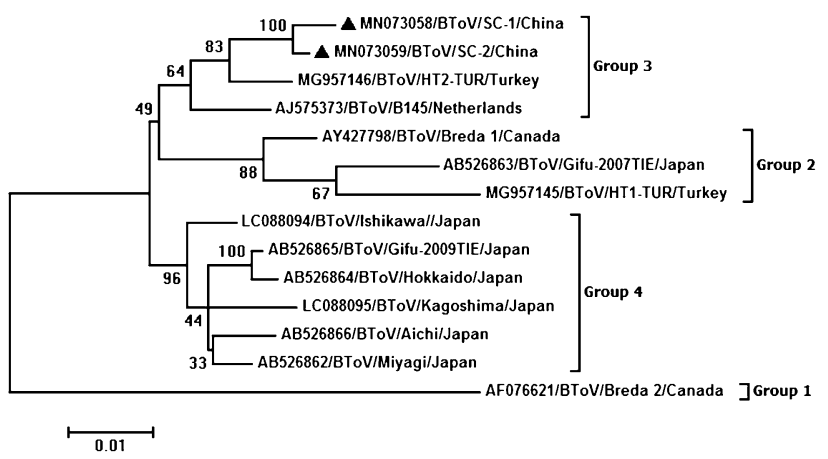

Fig. 2 Phylogenetic tree based on the deduced 1586-aa sequence of the complete $\mathrm{S}$ gene. Sequence alignments were performed using ClustalW in MEGA 7.0 software. The tree was constructed by the maximum-likelihood method with bootstrap values calculated for 1,000 replicates. The bovine torovirus strains investigated in this study are indicated by by black triangles

sequence identity) with the $12 \mathrm{BToV}$ S sequences available in the GenBank database. A phylogenetic analysis based on the complete amino acid sequence of the $\mathrm{S}$ protein showed that the BToVs could be separated into four groups (Fig. 2), designated tentatively as group 1 to group 4 . The two BToVs clustered with the BToV strains B145 and HT2 belonging to group 3 and were independently clustered in a small branch.

Further analysis showed that, compared to the 12 available BToV S sequences, strains SC- 1 and SC- 2 had 10 amino acid mutations, of which two were located in the signal peptide, six were located in the putative S1 region, and two were located in the putative $\mathrm{S} 2$ region. In addition, SC-1 had a 
continuous 7-amino-acid mutation at the end of the putative $\mathrm{S} 2$ region, and SC-2 had a unique amino acid mutation in the putative $\mathrm{S} 2$ region (Fig. 3).

\section{Complete HE gene sequences of strains SC-1 and SC-2}

The complete HE gene of strains SC- 1 and SC-2 are 1,260 bp in length and encode a protein of 419 amino acids. F-G-D$\mathrm{S}$, the putative esterase active site in all HE proteins, was located at aa positions 34-37. This complete HE gene shares 77.1-98.7\% nt sequence identity (76.2-98.1\% aa sequence identity) with all 16 available BToV HE gene sequences. Phylogenetic trees constructed using full-length HE gene sequences showed that the two HE sequences clustered into the same subgroup and belonged to genotype II (Fig. 4).

Furthermore, there was a unique amino acid variation (L202F) in strains SC-1 and SC-2 compared to the 16 available BToV HE sequences, and it is interesting that this mutation is located in the neutralizing epitope. In addition, the SC-1 strain had a unique amino acid variation (I289Y), and the SC-2 strain had three unique amino acid variations (K137S, S238Q, W290G) compared to the other available BToV HE sequences (Fig. 5).

\section{Recombination analysis}

BToV SC-1 and SC-2 were predicted to be recombination strains using the Recombination Detection Program (RDP 4.0) software, and standard similarity plot analysis was performed using Simplot 3.5.1 (Fig. 6). Notably, both strains SC-1 and SC-2 had the same predicted breakpoints in the crossover region, at nucleotide positions 20,810 and 28,047 in the 3' end of ORF1b and the 5' end of the $\mathrm{N}$ coding region, respectively.

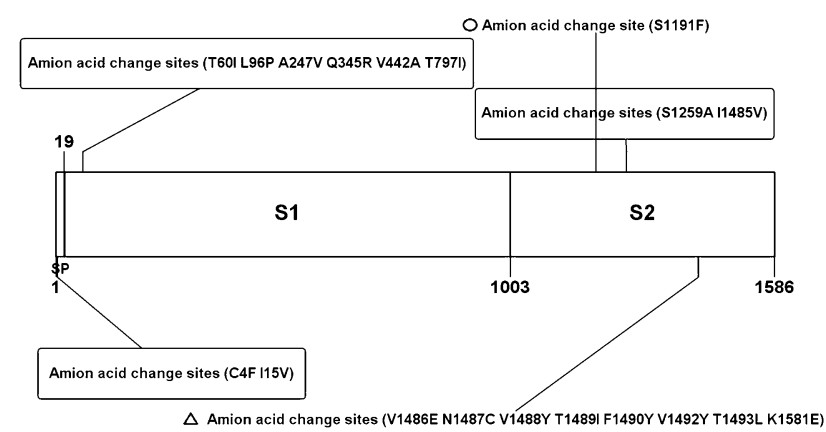

Fig. 3 Amino acid variations in the two complete $\mathrm{S}$ genes from this study. The numbers in the boxes indicate sites of identical amino acid changes in two strains in this study, compared to all complete BToV $\mathrm{S}$ protein in the GenBank database. The number indicated by a triangle is a unique variant of SC-1. The number indicated by a circle is a unique variant of SC-2

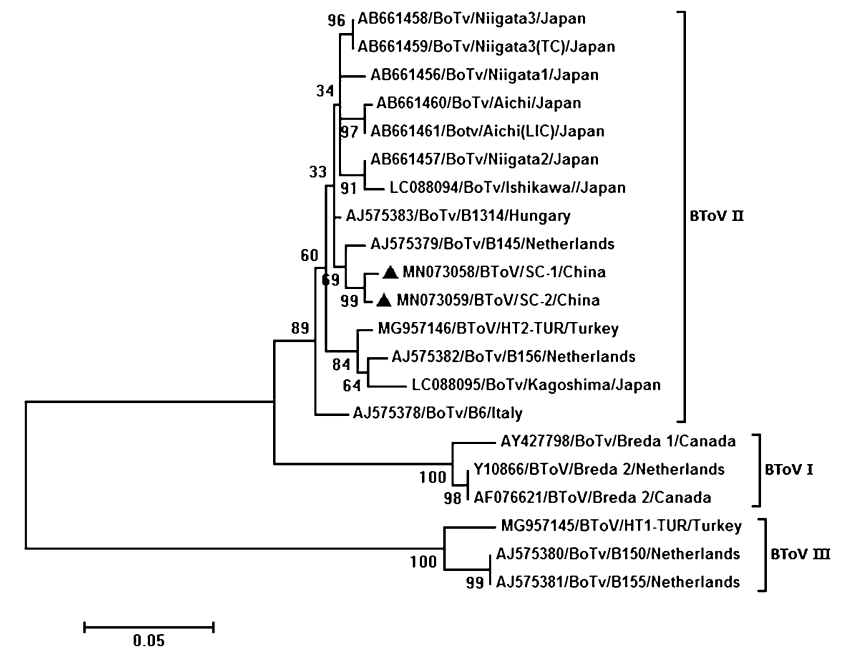

Fig. 4 Phylogenetic tree based on the deduced 419-aa sequence of the complete HE gene. Sequence alignments were performed using ClustalW in MEGA 7.0 software. The tree was constructed by the maximum-likelihood method with bootstrap values calculated for 1,000 replicates. The bovine torovirus strains investigated in this study are indicated by black triangles

\section{Discussion}

In this study, 21.73\% (20/92) of the diarrheic stool samples collected from dairy cows were found to be BToV positive. These samples were from four farms in three provinces, and the two more distant farms were separated by more than 1500 kilometers. The results indicate that BToV has circulated in Chinese cows with wide geographical

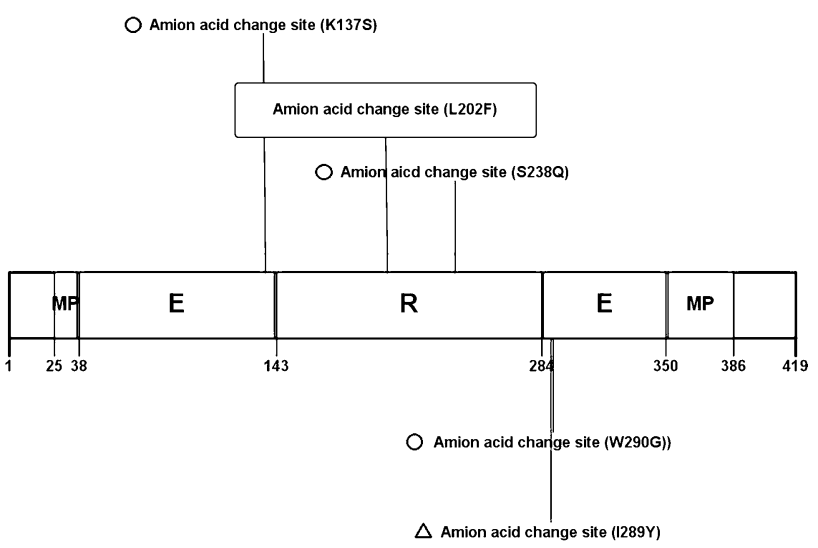

Fig. 5 Amino acid variants of the two complete HE genes from this study. The numbers in the boxes indicate identical amino acid change sites in two strains from this study compared to all HE sequences of $\mathrm{BToV}$ in the GenBank database. The number marked with a triangle is a unique variant of SC-1. The number marked with a circle is a unique variant of SC-2. E, esterase domain; MP, membrane-proximal domain; R, lectin domain 


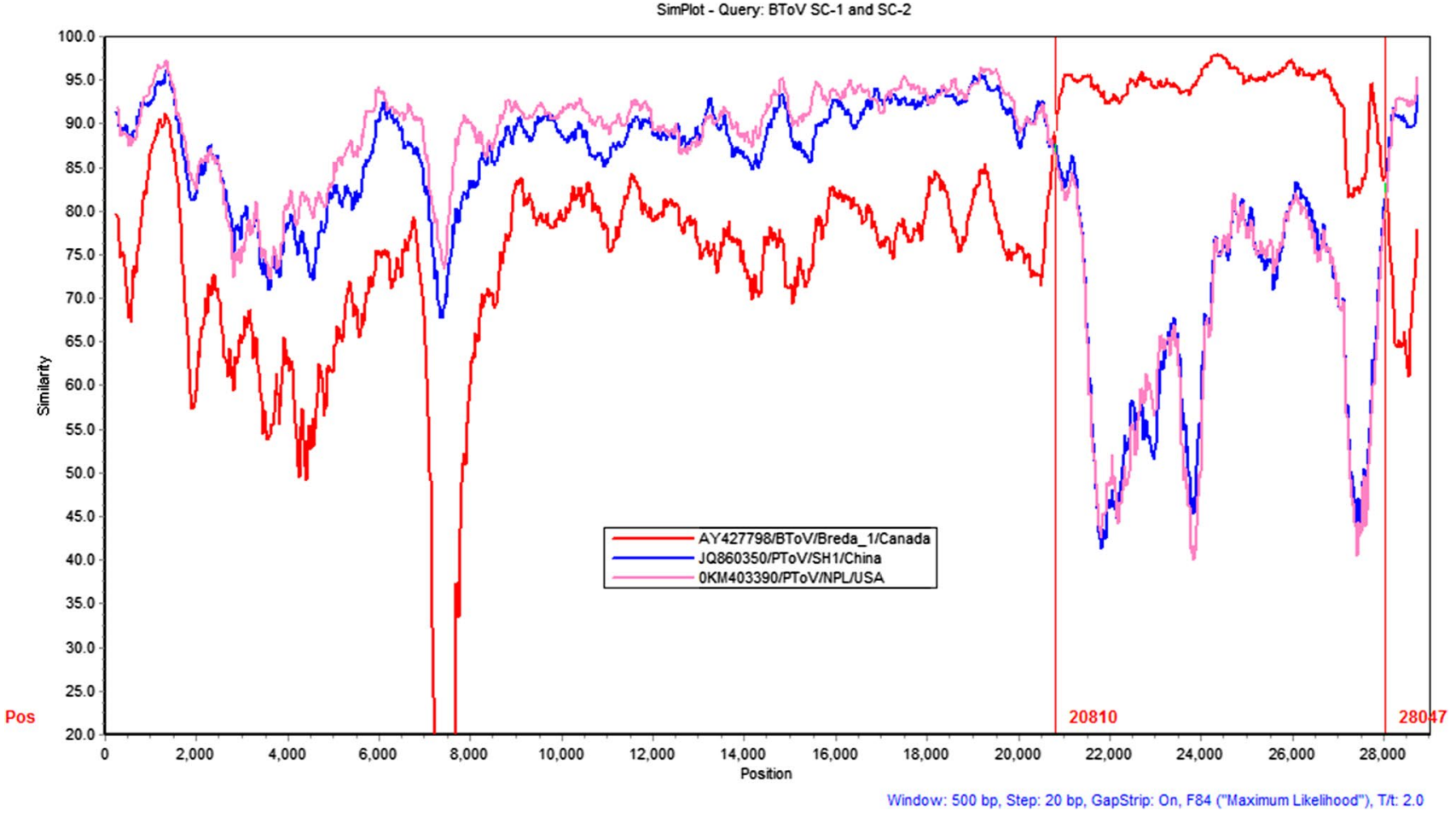

Fig. 6 Similarity plot analysis of the complete genome sequences of PToV SHI (blue line), BToV Breda (red line), and PToV NPL (pink line), with BToV SC-1 and SC-2 as query sequences, using a sliding window of $500 \mathrm{nt}$ and a moving step size of $20 \mathrm{nt}$

distribution. Although the number of samples was limited, the study confirmed the presence of BToV in China for the first time, which has significant implications for the diagnosis and control of diarrhea in calves in China. Moreover, it is worth noting that many BToV-positive samples were coinfected with other major intestinal viruses (Table 1), which is similar to the previous report from the USA [32]. This may lead to an increase in clinical severity and increased difficulty in diagnosing and controlling calf diarrhea.

In this research, we determined the obtained two complete genome sequences of two $\mathrm{BToV}$ isolates from the same farm in Sichuan province, increasing the number of $\mathrm{BToV}$ genome sequences in the GenBank database to five, thus contributing to a better understanding of the genome structure and genetic evolution of BToV. Phylogenetic analysis indicated that these two BToV isolates had a close genetic relationship to strains from Japan. Strains SC-1 and SC-2 were predicted to be recombinants derived from BToV strains Breda1 and PToV, in agreement with a previous report [8]. Moreover, the two Chinese strains shared identical unique amino acid changes in the $\mathrm{S}$ and HE genes when compared to the other strains with sequences available in the GenBank database, indicating the unique evolution in Chinese BToV strains. Interestingly, although these two isolates came from the same farm, their S and HE genes had their own unique amino acid variations. These results will be helpful for understanding the genetic evolution of BToV.

The $S$ protein is involved in the induction of neutralizing antibody production during the infection process and plays an important role in the pathogenesis of BToV, which is similar to that of coronaviruses [22, 24, 33]. Another member of the family Coronaviridae, $\mathrm{BCoV}$, has a genomic structure similar to that of $\mathrm{BToV}$, and mutations in the $\mathrm{S}$ protein are associated with viral antigenicity, pathogenicity, tissue tropism, and host range change [34, 35]. It has been reported that cleavage most likely occurs between amino acids 1,002 and 1,003 of the mature $\mathrm{S}$ protein in BToV, resulting in the formation of the S1 and S2 proteins [24]. The two Chinese strains shared two identical amino acid mutations in the signal peptide that might affect its function. It is worth noting that these two strains have six identical amino acid mutations in the putative S1 region. Since the S1 protein is responsible for receptor recognition and neutralizing antibody induction, mutations at amino acids 60 and 96 (T60I, L96P) might affect virus replication [36]. Therefore, the effects of these unique amino acid variations on the function of the proteins of strains SC-1 and SC-2 S is worth further study.

Based on the HE gene sequences, BTOV can be divided into three genotypes [18]. In this study, a phylogenetic tree constructed using all 18 complete HE gene sequences available in the GenBank database showed that the two HE 
sequences belonged to a genotype that is prevalent throughout the world [10, 12, 25, 37]. The torovirus HE protein contains three domains: MP, E and R, among which the $\mathrm{R}$ domain is involved in receptor recognition and plays an important role in the process of BToV infection [38]. The two Chinese strains shared an identical unique amino acid change in the $\mathrm{E}$ region. In addition, strain SC-2 had another unique amino acid change in the $\mathrm{E}$ region. Therefore, the ability of these mutations to affect viral receptor binding requires further investigation.

In conclusion, this study is the first to confirm the existence and prevalence of BTOV in Chinese calves with diarrhea, contributing to the diagnosis and control of calf diarrhea in China. Moreover, two complete BToV genome sequences were obtained from the clinical samples, and these two BToV isolates had unique amino acid changes in the $\mathrm{S}$ and HE proteins. These findings will enhance our understanding of the epidemic and the genetic evolution of BToV.

Acknowledgements This study was supported by funding from the 13th Five-Year Plan National Science and Technology Support Program (Grant number 2016YFD0500907).

\section{Compliance with ethical standards}

Conflict of interest The authors declare that they have no conflict of interest.

\section{References}

1. Cavanagh D (1997) Nidovirales: a new order comprising Coronaviridae and Arteriviridae. Arch Virol 142:629-633. https://doi. org/10.1007/978-1-4615-2996-5_39

2. Duckmanton L, Carman S, Nagy E, Petric M (1998) Detection of bovine torovirus in fecal specimens of calves with diarrhea from Ontario farms. J Clin Microbiol 36:1266-1270

3. Weiss M, Steck F, Kaderli R, Horzinek MC (1984) Antibodies to Berne virus in horses and other animals. Vet Microbiol 9:523531. https://doi.org/10.1016/0378-1135(84)90014-2

4. Kroneman A, Cornelissen LA, Horzinek MC, de Groot RJ, Egberink HF (1998) Identification and characterization of a porcine torovirus. J Virol 72:3507-3511

5. Beards GM, Hall C, Green J, Flewett TH, Lamouliatte F, Du Pasquier P (1984) An enveloped virus in stools of children and adults with gastroenteritis that resembles the Breda virus of calves. Lancet 1:1050-1052. https://doi.org/10.1016/s0140-6736(84)91454-5

6. Draker R, Roper RL, Petric M, Tellier R (2006) The complete sequence of the bovine torovirus genome. Virus Res 115:56-68. https://doi.org/10.1016/j.virusres.2005.07.005

7. Hoet AE, Cho KO, Chang KO, Loerch SC, Wittum TE, Saif LJ (2002) Enteric and nasal shedding of bovine torovirus (Breda virus) in feedlot cattle. Am J Vet Res 63:342-348. https://doi. org/10.2460/ajvr.2002.63.342

8. Ito T, Okada N, Okawa M, Fukuyama S, Shimizu M (2009) Detection and characterization of bovine torovirus from the respiratory tract in Japanese cattle. Vet Microbiol 136:366-371. https://doi. org/10.1016/j.vetmic.2008.11.014
9. Woode GN, Reed DE, Runnels PL, Herrig MA, Hill HT (1982) Studies with an unclassified virus isolated from diarrheic calves. Vet Microbiol 7:221-240. https://doi.org/10.1016/03781135(82)90036-0

10. Gulacti I, Isidan H, Sozdutmaz I (2014) Detection of bovine torovirus in fecal specimens from calves with diarrhea in Turkey. Arch Virol 159:1623-1627. https://doi.org/10.1007/s0070 5-014-1977-7

11. Haschek B, Klein D, Benetka V, Herrera C, Sommerfeld-Stur I, Vilcek S, Moestl K, Baumgartner W (2006) Detection of bovine torovirus in neonatal calf diarrhoea in Lower Austria and Styria (Austria). J Vet Med B Infect Dis Vet Public Health 53:160-165. https://doi.org/10.1111/j.1439-0450.2006.00936.x

12. Kirisawa R, Takeyama A, Koiwa M, Iwai H (2007) Detection of bovine torovirus in fecal specimens of calves with diarrhea in Japan. J Vet Med Sci 69:471-476. https://doi.org/10.1292/ jvms.69.471

13. Liebler EM, Klüver S, Pohlenz J, Koopmans M (1992) The significance of bredavirus as a diarrhea agent in calf herds in Lower Saxony. Dtw Deutsche Tierärztliche Wochenschrift 99:195-200

14. Lojkic I, Kresic N, Simic I, Bedekovic T (2015) Detection and molecular characterisation of bovine corona and toroviruses from Croatian cattle. BMC Vet Res 11:202. https://doi.org/10.1186/ s12917-015-0511-9

15. Nogueira JS, Asano KM, de Souza SP, Brandao PE, Richtzenhain LJ (2013) First detection and molecular diversity of Brazilian bovine torovirus (BToV) strains from young and adult cattle. Res Vet Sci 95:799-801. https://doi.org/10.1016/j.rvsc.2013.04.006

16. Park SJ, Oh EH, Park SI, Kim HH, Jeong YJ, Lim GK, Hyun BH, Cho KO (2008) Molecular epidemiology of bovine toroviruses circulating in South Korea. Vet Microbiol 126:364-371. https:// doi.org/10.1016/j.vetmic.2007.07.012

17. Pérez E, Kummeling A, Janssen MMH, Jiménez C, Alvarado R, Caballero M, Donado P, Dwinger RH (1998) Infectious agents associated with diarrhoea of calves in the canton of Tilarán. Costa Rica. 33:195-205. https://doi.org/10.1016/s0167-5877(97)00038 $-\mathrm{X}$

18. Smits SL, Lavazza A, Matiz K, Horzinek MC, Koopmans MP, de Groot RJ (2003) Phylogenetic and evolutionary relationships among torovirus field variants: evidence for multiple intertypic recombination events. J Virol 77:9567-9577. https://doi. org/10.1128/jvi.77.17.9567-9577.2003

19. Vorster JH, Gerdes GH (1993) Breda virus-like particles in calves in South Africa. J S Afr Vet Assoc 64:58

20. Sun H, Lan D, Lu L, Chen M, Wang C, Hua X (2014) Molecular characterization and phylogenetic analysis of the genome of porcine torovirus. Arch Virol 159(4):773-778. https://doi. org/10.1007/s00705-013-1861-x

21. Hoet AE, Saif LJ (2004) Bovine torovirus (Breda virus) revisited. Anim Health Res Rev 5:157-171. https://doi.org/10.1079/ahr20 0498

22. Li F (2016) Structure, function, and evolution of coronavirus spike proteins. Annu Rev Virol 3:237-261. https://doi.org/10.1146/ annurev-virology-110615-042301

23. Langereis MA, Zeng Q, Gerwig GJ, Frey B, von Itzstein M, Kamerling JP, de Groot RJ, Huizinga EG (2009) Structural basis for ligand and substrate recognition by torovirus hemagglutinin esterases. Proc Natl Acad Sci USA 106:15897-15902. https://doi. org/10.1073/pnas.0904266106

24. Duckmanton LM, Tellier R, Liu P, Petric M (1998) Bovine torovirus: sequencing of the structural genes and expression of the nucleocapsid protein of Breda virus. Virus Res 58:83-96. https:// doi.org/10.1016/s0168-1702(98)00104-X

25. Hurst KR, Ye R, Goebel SJ, Jayaraman P, Masters PS (2010) An interaction between the nucleocapsid protein and a component of the replicase-transcriptase complex is crucial for the infectivity 
of coronavirus genomic RNA. J Virol 84:10276-10288. https:// doi.org/10.1128/JVI.01287-10

26. Cui J, Fu X, Xie J, Gao M, Hong M, Chen Y, Su S, Li S (2014) Critical role of cellular cholesterol in bovine rotavirus infection. Virol J 11:98. https://doi.org/10.1186/1743-422X-11-98

27. Gong X, Liu L, Zheng F, Chen Q, Li Z, Cao X, Yin H, Zhou J, Cai X (2014) Molecular investigation of bovine viral diarrhea virus infection in yaks (Bos gruniens) from Qinghai, China. Virol J 11:29. https://doi.org/10.1186/1743-422X-11-29

28. Guo Z, He Q, Zhang B, Yue H, Tang C (2019) Detection and molecular characteristics of neboviruses in dairy cows in China. J Gen Virol 100:35-45. https://doi.org/10.1099/jgv.0.001172

29. Keha A, Xue L, Yan S, Yue H, Tang C (2019) Prevalence of a novel bovine coronavirus strain with a recombinant hemagglutinin/esterase gene in dairy calves in China. Transbound Emerg Dis 66:1971-1981. https://doi.org/10.1111/tbed.13228

30. Wang Y, Yue H, Tang C (2019) Prevalence and complete genome of bovine norovirus with novel VP1 genotype in calves in China. Sci Rep 9:12023. https://doi.org/10.1038/s41598-019-48569-4

31. Smiley JR, Hoet AE, Tråvén M, Tsunemitsu H, Saif LJ (2003) Reverse transcription-PCR assays for detection of bovine enteric caliciviruses (BEC) and analysis of the genetic relationships among BEC and human caliciviruses. J Clin Microbiol 41:3089_ 3099. https://doi.org/10.1128/jcm.41.7.3089-3099.2003

32. Cho YI, Han JI, Wang C, Cooper V, Schwartz K, Engelken T, Yoon KJ (2013) Case-control study of microbiological etiology associated with calf diarrhea. Vet Microbiol 166:375-385. https ://doi.org/10.1016/j.vetmic.2013.07.001

33. Yoo D, Deregt D (2001) A single amino acid change within antigenic domain II of the spike protein of bovine coronavirus confers resistance to virus neutralization. Clin Diagn Lab Immunol 8:297. https://doi.org/10.1128/CDLI.8.2.297-302.2001

34. Gallagher TM, Buchmeier MJ (2001) Coronavirus spike proteins in viral entry and pathogenesis. Virology 279:371-374. https:// doi.org/10.1006/viro.2000.0757

35. Peng G, Xu L, Lin YL, Chen L, Pasquarella JR, Holmes KV, Li F (2012) Crystal structure of bovine coronavirus spike protein lectin domain. J Biol Chem 287:41931-41938. https://doi.org/10.1074/ jbc.M112.418210

36. Ito T, Katayama S, Okada N, Masubuchi K, Si Fukuyama, Shimizu M (2012) Genetic and antigenic characterization of newly isolated bovine toroviruses from Japanese cattle. J Clin Microbiol 48:1795-1800. https://doi.org/10.1128/JCM.02339-09

37. Aita T, Kuwabara M, Murayama K, Sasagawa Y, Yabe S, Higuchi R, Tamura T, Miyazaki A, Tsunemitsu H (2012) Characterization of epidemic diarrhea outbreaks associated with bovine torovirus in adult cows. Arch Virol 157:423-431. https://doi.org/10.1007/ s00705-011-1183-9

38. Cornelissen LA, Wierda CM, Fj VDM, Herrewegh AA, Horzinek MC, Egberink HF, de Groot RJ (1997) Hemagglutinin-esterase, a novel structural protein of torovirus. J Virol 71:5277-5286

Publisher's Note Springer Nature remains neutral with regard to jurisdictional claims in published maps and institutional affiliations. 